\title{
A new systolic parameter defined as the ratio of brachial pre-ejection period to brachial ejection time predicts overall and cardiovascular mortality in hemodialysis patients
}

\begin{abstract}
Szu-Chia Chen ${ }^{1,2}$, Jer-Ming Chang ${ }^{1,2,3}$, Jer-Chia Tsai ${ }^{1,3}$, Po-Chao Hsu ${ }^{4}$, Tsung-Hsien Lin $^{4,5}$, Ho-Ming Su${ }^{2,4,5}$, Wen-Chol Voon ${ }^{4,5}$, Shang-Jyh Hwang ${ }^{1,3}$ and Hung-Chun Chen ${ }^{1,3}$

Impaired left ventricular systolic function is an important cause of mortality in hemodialysis patients. An increase in the ratio of pre-ejection period (PEP) to ejection time (ET) is associated with a decrease in left ventricular systolic function. Brachial PEP (bPEP) and brachial ET (bET) can be automatically determined from an ankle-brachial index (ABI)-form device. The aim of this study was to investigate whether bPEP/bET was a useful predictor for overall and cardiovascular mortality in hemodialysis patients. We enrolled 212 hemodialysis patients in one regional hospital. The bPEP and bET were measured using an ABI-form device. The mean follow-up period was $\mathbf{2 8 . 3} \pm 5.7$ months. The relative mortality risk was analyzed by Cox-regression methods. Twenty-two deaths were recorded in 212 patients (10.4\%). In a multivariate analysis, the bPEP/bET (hazard ratio [HR], 1.055; $P=0.047)$ and serum creatinine level $(P=0.029)$ were positively and negatively associated with overall mortality, respectively. In addition, increased bPEP/bET (HR, 1.080; $P=0.017)$, increased fasting glucose $(P=0.046)$ and decreased serum creatinine level $(P=0.004)$ were independent predictors for cardiovascular mortality. Our findings show that $\mathrm{bPEP} / \mathrm{bET}$, a surrogate of left ventricular systolic function, is a useful predictor for overall and cardiovascular mortality in hemodialysis patients. Screening hemodialysis patients by means of bPEP/bET may help to identify a high-risk group for increased mortality. Hypertension Research (2010) 33, 492-498; doi:10.1038/hr.2010.24; published online 5 March 2010
\end{abstract}

Keywords: brachial ejection time; brachial pre-ejection period; cardiovascular mortality; hemodialysis; overall mortality

\section{INTRODUCTION}

End-stage renal disease is an increasing worldwide public health problem associated with increased morbidity and mortality. ${ }^{1,2}$ Cardiovascular disease is the leading cause of mortality in hemodialysis patients. Systolic time intervals are an established noninvasive technique for the quantitative assessment of cardiac performance and remain valuable for clinical application. ${ }^{3}$ Prolonged pre-ejection period (PEP) and shortened ejection time (ET) have been reported to be significantly correlated with decreased left ventricular systolic function. ${ }^{3-5}$ A high correlation $(r=-0.90)$ between PEP/ET and left ventricular ejection fraction has been shown in patients with a wide variety of heart disease. ${ }^{4}$ However, PEP and ET are frequently obtained from echocardiography, which may preclude their application in evaluating left ventricular systolic function if echocardiography is not available. A clinical device, ankle-brachial index
(ABI)-form (Colin VP1000, Komaki, Japan), has been developed to automatically and simultaneously measure blood pressures in both arms and ankles and record pulse waves of the brachial and posterior tibial arteries, using an automated oscillometric method. Using this device, we can easily and automatically calculate the brachial PEP (bPEP) and brachial ET (bET) by analyzing the signals of ECG, phonocardiogram and brachial pressure volume waveform. ${ }^{6,7}$ Recently, we have shown bPEP/bET to have significant correlation with left ventricular ejection fraction and it is a useful parameter in prediction of impaired left ventricular systolic function. ${ }^{6}$ However, no study has evaluated the relationship between bPEP/bET and clinical outcome in hemodialysis patients. The aim of this study was to investigate whether $\mathrm{bPEP} / \mathrm{bET}$ was a useful predictor of overall and cardiovascular mortality in patients receiving hemodialysis.

\footnotetext{
${ }^{1}$ Division of Nephrology, Department of Internal Medicine, Kaohsiung Medical University Hospital, Kaohsiung Medical University, Kaohsiung, Taiwan, ROC; ${ }^{2}$ Department of Internal Medicine, Kaohsiung Municipal Hsiao-Kang Hospital, Kaohsiung Medical University, Kaohsiung, Taiwan, ROC; ${ }^{3}$ Faculty of Renal Care, Kaohsiung Medical University, Kaohsiung, Taiwan, ROC; ${ }^{4}$ Division of Cardiology, Department of Internal Medicine, Kaohsiung Medical University Hospital, Kaohsiung Medical University, Kaohsiung, Taiwan, ROC and ${ }^{5}$ Faculty of Medicine, College of Medicine, Kaohsiung Medical University, Kaohsiung, Taiwan, ROC

Correspondence: Dr H-M Su, Department of Internal Medicine, Kaohsiung Municipal Hsiao-Kang Hospital, Kaohsiung Medical University, 482, Shan-Ming Road, Hsiao-Kang District, Kaohsiung 812, Taiwan, ROC.

E-mail: cobeshm@seed.net.tw
}

Received 21 November 2009; revised 14 January 2010; accepted 26 January 2010; published online 5 March 2010 


\section{METHODS}

\section{Study patients and design}

The study was conducted at one dialysis clinic in a regional hospital in Taiwan. All routine hemodialysis patients in this hospital were included except 6 patients refusing ABI-form device examinations, 4 patients with atrial fibrillation and 13 patients with inadequate image visualization. Ultimately, 212 patients (95 males and 117 females) formed our study group. There was no patient with complete left bundle branch block in this study. The protocol was approved by our Institutional Review Board and all enrolled patients gave written, informed consent.

\section{Hemodialysis}

All patients underwent routine hemodialysis three times a week using a Toray 321 machine (Toray Medical Company, Tokyo, Japan). Each hemodialysis session was performed for $3-4 \mathrm{~h}$ using a dialyzer with a blood flow rate of 250-300 $\mathrm{ml} \mathrm{min}^{-1}$ and dialysate flow of $500 \mathrm{ml} \mathrm{min}^{-1}$.

\section{Assessment of bPEP, bET, ABI and brachial-ankle pulse wave velocity}

The values of bPEP and bET were measured $10-30 \mathrm{~min}$ before hemodialysis. The bPEP and bET were measured using an ABI-form device, which automatically and simultaneously measures blood pressures in both arms and ankles using an oscillometric method. ${ }^{7-9}$ Occlusion and monitoring cuffs were placed tightly around the upper arm without blood access and both sides of the lower extremities in the supine position. The bET was automatically measured from the foot to the dicrotic notch (equivalent to the incisure on the downstroke of the aortic pressure wave contour produced by the closure of the aortic valve) of the pulse volume waveform. Total electromechanical systolic interval $\left(\mathrm{QS}_{2}\right)$ was measured from the onset of the QRS complex on the ECG to the first high-frequency vibrations of the aortic component of the second heart sound on the phonocardiogram. The bPEP was also automatically calculated by subtracting the bET from the $\mathrm{QS}_{2}$ (Figure 1). In this study, the bPEP and bET were obtained from the right and left brachia in 168 and 44 patients, respectively. The values of $\mathrm{ABI}$ and brachial-ankle pulse wave velocity were also measured, and the measurement method has been reported and validated in earlier studies. ${ }^{7-9}$

\section{Collection of demographic, medical and laboratory data}

Demographic and medical data, including age, gender, smoking history (ever vs. never) and comorbid conditions, were obtained from medical records and interviews with patients. The body mass index was calculated as the ratio of weight in kilograms divided by the square of height in meters. Laboratory data were measured from fasting blood samples using an autoanalyzer (COBAS Integra 400; Roche Diagnostics GmbH, Mannheim, Germany). High-sensitivity C-reactive protein (Dade Behring Marburg GmbH, Marburg, Germany) was

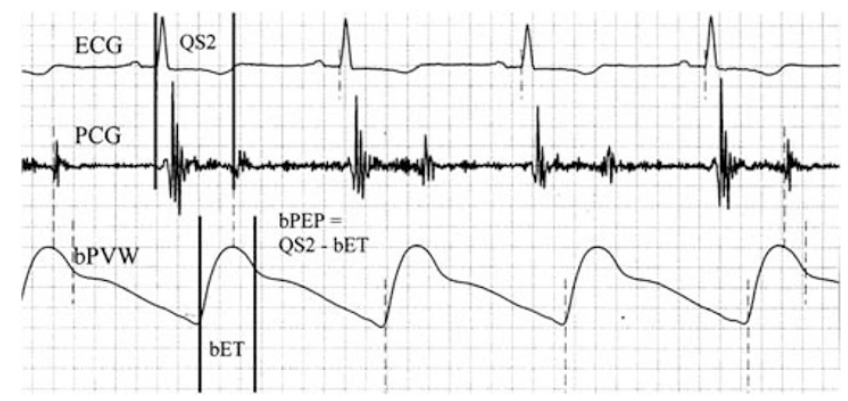

Figure 1 The brachial ejection time (bET) was automatically measured from the foot to the dicrotic notch of the brachial pulse volume waveform (bPVW). Total electromechanical systolic interval $\left(\mathrm{QS}_{2}\right)$ was measured from the onset of the QRS complex on the ECG to the first high-frequency vibrations of the aortic component of the second heart sound on the phonocardiogram (PCG). The brachial pre-ejection period (bPEP) was also automatically calculated by subtracting the $\mathrm{bET}$ from the $\mathrm{QS}_{2}$. measured by commercially available kits. Serum intact parathyroid hormone (PTH) concentration was evaluated using a commercially available two-sided immunoradiometric assay (CIS bio international, France). Blood samples were obtained within 1 month of enrollment. Kt/V was evaluated monthly as a marker of dialysis efficiency and was determined according to the Gotch procedure. ${ }^{10}$ In addition, information regarding patient medications including aspirin, angiotensin-converting enzyme inhibitors (ACEI), angiotensin II receptor blockers (ARB) and HMG-CoA reductase inhibitors (statins) during the study period was obtained from medical records.

\section{Statistical analysis}

Statistical analysis was performed using SPSS 12.0 for Windows (SPSS Inc., Chicago, IL, USA). Data are expressed as percentages or means \pm s.d. The differences between groups were checked by $\chi^{2}$ test for categorical variables or by independent $t$-test for continuous variables. The relationship between two continuous variables was assessed by a bivariate correlation method (Pearson's correlation). Time to death and covariates of risk factors were modeled using the Cox proportional hazards model. Age, gender and significant variables in the univariate analysis were further analyzed by multivariate analysis. A $P$-value $<0.05$ was considered significant.

\section{RESULTS}

The clinical characteristics of study patients are shown in Table 1 . The mean age of the 212 patients was $59.3 \pm 13.1$ years. The bPEP/bET was $0.35 \pm 0.08$. The prevalence of heart failure was $17.0 \%$. The differences between patients in terms of survival vs. mortality were shown in Table 2. Compared with survival-group patients, patients in the mortality group were significantly associated with shorter duration of hemodialysis, higher prevalence of a history of coronary artery disease, higher bPEP/bET and lower creatinine and PTH levels. In addition, patients in the mortality group had a higher percentage of having received aspirin or ACEI and/or ARB therapy.

We performed further analysis to identify the determinants of duration of hemodialysis and found that age $(r=-0.172 ; P=0.012)$ was negatively correlated with the duration of hemodialysis. In addition, patients with diabetes mellitus $(P<0.001)$ and hypertension $(P=0.020)$ had shorter durations of dialysis.

In addition, the use of aspirin or ACEI and/or ARB was more frequent among patients with coronary artery disease $(26.7 \%$ vs. $6.6 \%$, $P<0.001 ; 31.7 \%$ vs. $15.2 \% ; P=0.007$, respectively). Patients with diabetes mellitus and hypertension had a higher percentage of having received statins and ACEI and/or ARB therapy, respectively $(39.0 \%$ vs. $22.5 \% ; P=0.010 ; 27.3 \%$ vs. $1.6 \%, P<0.001)$.

The mean follow-up period was $28.3 \pm 5.7$ months. During the period of follow-up, 22 deaths were recorded in these 212 patients (10.4\%), including fatal cardiovascular events $(n=15)$, malignancy $(n=3)$, infectious disease $(n=2)$, gastrointestinal bleeding $(n=1)$ and others $(n=1)$. Table 3 shows a Cox proportional hazards-regression analysis for overall mortality. The univariate regression analysis shows that the hazard ratio (HR) of the bPEP/bET was $1.051 \quad(95 \%$ confidence interval, $1.005-1.100 ; P=0.030)$. In addition, other variables including the presence of coronary artery disease, short duration of hemodialysis, increased fasting glucose, decreased serum creatinine level and the use of aspirin or ACEI and/or ARB were associated with a significant increase in overall mortality. In the multivariate analysis, the bPEP/bET (HR, 1.055; 95\% confidence interval, 1.001-1.112; $P=0.047)$ was positively associated with and serum creatinine level (HR, $0.733 ; P=0.029$ ) was negatively associated with overall mortality.

Fifteen cardiovascular deaths were documented during the followup period, including heart failure $(n=8)$, myocardial infarction $(n=4)$, cerebral infarction $(n=2)$ and ventricular fibrillation $(n=1)$. A Cox proportional hazards-regression analysis for cardiovascular mortality 
Table 1 The characteristics of the study patients

\begin{tabular}{|c|c|}
\hline Characteristics & All patients $(\mathrm{n}=212)$ \\
\hline Age (year) & $59.3 \pm 13.1$ \\
\hline Male gender (\%) & 44.8 \\
\hline Duration of dialysis (month) & $74.7 \pm 47.8$ \\
\hline Smoking history (\%) & 25.5 \\
\hline Diabetes mellitus (\%) & 39.2 \\
\hline Hypertension (\%) & 71.2 \\
\hline Coronary artery disease (\%) & 28.8 \\
\hline Cerebrovascular disease (\%) & 9.4 \\
\hline Systolic BP (mm Hg) & $145.5 \pm 25.1$ \\
\hline Diastolic BP (mm Hg) & $79.3 \pm 15.4$ \\
\hline Pulse pressure $(\mathrm{mm} \mathrm{Hg})$ & $66.2 \pm 17.3$ \\
\hline Body mass index $\left(\mathrm{kg} \mathrm{m}^{-2}\right)$ & $23.9 \pm 3.6$ \\
\hline $\mathrm{ABI}<0.9(\%)$ & 13.6 \\
\hline $\mathrm{baPWV}\left(\mathrm{cm} \mathrm{s}^{-1}\right)$ & $1917.5 \pm 543.7$ \\
\hline $\mathrm{bPEP}(\mathrm{ms})$ & $97.1 \pm 18.7$ \\
\hline $\mathrm{bET}(\mathrm{ms})$ & $284.0 \pm 29.1$ \\
\hline $\mathrm{bPEP} / \mathrm{bET}$ & $0.35 \pm 0.08$ \\
\hline \multicolumn{2}{|l|}{ Laboratory parameters } \\
\hline Albumin (g per $100 \mathrm{ml}$ ) & $3.8 \pm 0.3$ \\
\hline Fasting glucose (mg per $100 \mathrm{ml}$ ) & $120.4 \pm 55.2$ \\
\hline Triglyceride (mg per $100 \mathrm{ml}$ ) & $171.4 \pm 129.0$ \\
\hline Cholesterol (mg per $100 \mathrm{ml}$ ) & $184.0 \pm 41.9$ \\
\hline HDL cholesterol (mg per $100 \mathrm{ml}$ ) & $46.5 \pm 14.7$ \\
\hline LDL cholesterol (mg per $100 \mathrm{ml}$ ) & $88.0 \pm 26.5$ \\
\hline Creatinine (mg per $100 \mathrm{ml}$ ) & $10.3 \pm 2.3$ \\
\hline Hematocrit (\%) & $30.7 \pm 3.3$ \\
\hline Calcium (mg per $100 \mathrm{ml}$ ) & $9.8 \pm 0.8$ \\
\hline Phosphate (mg per $100 \mathrm{ml}$ ) & $4.9 \pm 1.2$ \\
\hline Uric acid (mg per $100 \mathrm{ml}$ ) & $7.6 \pm 1.5$ \\
\hline PTH $\left(\mathrm{pg} \mathrm{m}^{-1}\right)$ & $501.6 \pm 429.2$ \\
\hline $\operatorname{hsCRP}\left(\mathrm{mgl}^{-1}\right)$ & $0.8 \pm 1.6$ \\
\hline $\mathrm{Kt} / \mathrm{V}$ & $1.3 \pm 0.2$ \\
\hline Cardiothoracic ratio $>50 \%$ & 44.8 \\
\hline \multicolumn{2}{|l|}{ Medications } \\
\hline Aspirin use (\%) & 12.3 \\
\hline ACEI and/or ARB use (\%) & 19.8 \\
\hline Statins use (\%) & 28.8 \\
\hline
\end{tabular}

Abbreviations: $\mathrm{ABI}$, ankle-brachial index; $\mathrm{ACEI}$, angiotensin-converting enzyme inhibitor; ARB, angiotensin II receptor blocker; baPWV, brachial-ankle pulse wave velocity; BP, blood pressure; bPEP, brachial pre-ejection period; bET, brachial ejection time; HDL, high-density lipoprotein; hsCRP, high-sensitivity C-reactive protein; LDL, low-density lipoprotein; PTH, parathyroid hormone.

is shown in Table 4. In the multivariate analysis, increased bPEP/bET (HR, 1.080; $P=0.017)$, increased fasting glucose $(\mathrm{HR}, 1.007 ; P=0.046)$ and decreased serum creatinine level $(\mathrm{HR}, 0.610 ; P=0.004)$ were independent predictors for cardiovascular mortality.

Fifteen patients were New York Heart Association class III and IV, and 21 patients were New York Heart Association class I and II. We performed further forward multivariate analysis after including congestive heart failure as a variable and found that the presence of congestive heart failure (HR, 7.798; $P<0.001$ and HR, 10.255; $P<0.001$, respectively) and increased bPEP/bET (HR, 1.056; $P=0.029$ and $\mathrm{HR}, 1.063 ; P=0.017$, respectively) were independent predictors of overall and cardiovascular mortality.

We further performed survival analysis after excluding the value of serum creatinine and found that there was still an independent
Table 2 Comparison of baseline characteristics between patients with survival and mortality

\begin{tabular}{|c|c|c|c|}
\hline Characteristics & $\begin{array}{l}\text { Patients with } \\
\text { survival } \\
(\mathrm{n}=190)\end{array}$ & $\begin{array}{c}\text { Patients with } \\
\text { mortality } \\
(\mathrm{n}=22)\end{array}$ & $\mathrm{P}$ \\
\hline Age (year) & $58.9 \pm 13.2$ & $63.3 \pm 11.6$ & 0.130 \\
\hline Male gender (\%) & 44.7 & 45.5 & 0.949 \\
\hline Duration of dialysis (month) & $77.0 \pm 48.7$ & $55.2 \pm 34.2$ & 0.043 \\
\hline Smoking history (\%) & 24.2 & 36.4 & 0.216 \\
\hline Diabetes mellitus (\%) & 37.4 & 54.5 & 0.118 \\
\hline Hypertension (\%) & 70.0 & 81.8 & 0.246 \\
\hline Coronary artery disease (\%) & 25.8 & 54.5 & 0.005 \\
\hline Cerebrovascular disease (\%) & 8.4 & 18.2 & 0.137 \\
\hline Systolic BP (mm Hg) & $145.97 \pm 24.4$ & $142.2 \pm 30.7$ & 0.517 \\
\hline Diastolic BP (mm Hg) & $79.8 \pm 14.6$ & $75.7 \pm 21.1$ & 0.387 \\
\hline Pulse pressure $(\mathrm{mm} \mathrm{Hg})$ & $66.1 \pm 17.6$ & $66.6 \pm 14.6$ & 0.918 \\
\hline Body mass index $\left(\mathrm{kg} \mathrm{m}^{-2}\right)$ & $23.9 \pm 3.6$ & $23.0 \pm 3.9$ & 0.293 \\
\hline$A B \mid<0.9(\%)$ & 13.2 & 22.7 & 0.223 \\
\hline $\mathrm{baPWV}\left(\mathrm{cm} \mathrm{s}^{-1}\right)$ & $1912.4 \pm 540.7$ & $1961.2 \pm 580.6$ & 0.691 \\
\hline bPEP (ms) & $96.4 \pm 18.4$ & $102.4 \pm 21.4$ & 0.161 \\
\hline $\mathrm{bET}(\mathrm{ms})$ & $284.9 \pm 28.9$ & $275.4 \pm 29.5$ & 0.146 \\
\hline $\mathrm{bPEP} / \mathrm{bET}$ & $0.34 \pm 0.08$ & $0.38 \pm 0.11$ & 0.034 \\
\hline \multicolumn{4}{|l|}{ Laboratory parameters } \\
\hline Albumin (g per $100 \mathrm{ml}$ ) & $3.8 \pm 0.3$ & $3.8 \pm 0.4$ & 0.190 \\
\hline Fasting glucose (mg per $100 \mathrm{ml}$ ) & $117.9 \pm 52.6$ & $145.4 \pm 73.0$ & 0.124 \\
\hline Triglyceride (mg per $100 \mathrm{ml}$ ) & $173.6 \pm 132.5$ & $152.6 \pm 93.2$ & 0.469 \\
\hline Cholesterol (mg per $100 \mathrm{ml}$ ) & $185.3 \pm 41.5$ & $173.0 \pm 44.3$ & 0.192 \\
\hline HDL cholesterol (mg per $100 \mathrm{ml}$ ) & $45.6 \pm 16.2$ & $46.5 \pm 14.6$ & 0.801 \\
\hline LDL cholesterol (mg per $100 \mathrm{ml}$ ) & $88.9 \pm 27.1$ & $79.0 \pm 17.7$ & 0.121 \\
\hline Creatinine (mg per $100 \mathrm{ml}$ ) & $10.4 \pm 2.2$ & $9.0 \pm 2.7$ & 0.005 \\
\hline Hematocrit (\%) & $30.6 \pm 3.3$ & $31.4 \pm 3.7$ & 0.297 \\
\hline Calcium (mg per $100 \mathrm{ml}$ ) & $9.8 \pm 0.9$ & $9.7 \pm 0.9$ & 0.724 \\
\hline Phosphate (mg per $100 \mathrm{ml}$ ) & $4.9 \pm 1.2$ & $4.8 \pm 1.2$ & 0.887 \\
\hline Uric acid (mg per $100 \mathrm{ml}$ ) & $7.7 \pm 1.6$ & $7.3 \pm 1.2$ & 0.303 \\
\hline PTH $\left(p g \mathrm{ml}^{-1}\right)$ & $520.5 \pm 440.6$ & $330.7 \pm 256.4$ & 0.006 \\
\hline $\operatorname{hsCRP}\left(\mathrm{mgl}^{-1}\right)$ & $0.7 \pm 1.5$ & $1.2 \pm 2.0$ & 0.262 \\
\hline $\mathrm{Kt} / \mathrm{V}$ & $1.3 \pm 0.2$ & $1.3 \pm 0.3$ & 0.922 \\
\hline Cardiothoracic ratio $>50 \%$ & 44.2 & 50.0 & 0.605 \\
\hline \multicolumn{4}{|l|}{ Medications } \\
\hline Aspirin use (\%) & 10.6 & 27.3 & 0.024 \\
\hline ACEI and/or ARB use (\%) & 18.0 & 36.4 & 0.041 \\
\hline Statins use (\%) & 30.7 & 13.6 & 0.135 \\
\hline
\end{tabular}

Abbreviations: $A B I$, ankle-brachial index; $A C E I$, angiotensin-converting enzyme inhibitor; ARB, angiotensin II receptor blocker; baPWV, brachial-ankle pulse wave velocity; BP, blood pressure bPEP, brachial pre-ejection period; bET, brachial ejection time; HDL, high-density lipoprotein: hsCRP, high-sensitivity C-reactive protein; LDL, low-density lipoprotein; PTH, parathyroid hormone.

association between bPEP/bET and both overall and cardiovascular mortality (HR, $1.065 ; P=0.022$ and $\mathrm{HR}, 1.105 ; P=0.002$, respectively). In addition, there was no significant correlation between $\mathrm{bPEP} / \mathrm{bET}$ and serum creatinine level $(r=0.107 ; P=0.120)$.

\section{Reproducibility}

The mean percentage errors for bPEP, bET and bPEP/bET measurement $(3.6 \pm 3.6,2.0 \pm 1.5$ and $4.2 \pm 4.4 \%$, respectively) have been reported in our earlier study. 6 
Table 3 Predictors for overall mortality using Cox proportional hazards model

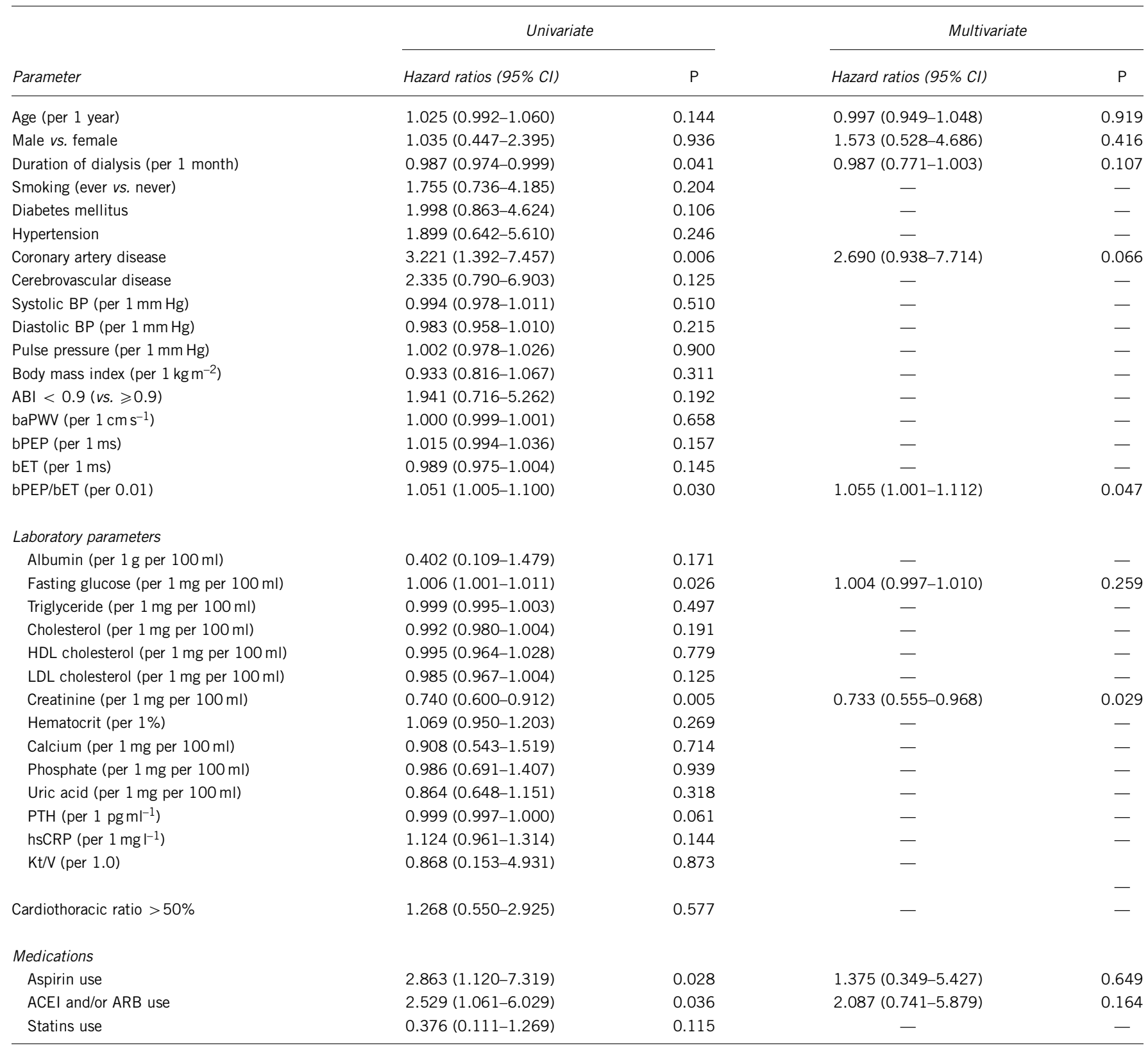

Abbreviations: ABI, ankle-brachial index; ACEI, angiotensin-converting enzyme inhibitor; ARB, angiotensin II receptor blocker; baPWV, brachial-ankle pulse wave velocity; BP, blood pressure; bPEP, brachial pre-ejection period; bET, brachial ejection time; $\mathrm{Cl}$, confidence interval; HDL, high-density lipoprotein; hsCRP, high-sensitivity C-reactive protein; LDL, low-density lipoprotein; PTH, parathyroid hormone.

Values express as hazard ratios and $95 \% \mathrm{Cl}$.

\section{DISCUSSION}

In this study, we evaluated the predictors for overall and cardiovascular mortality in 212 patients receiving hemodialysis. We found that the $\mathrm{bPEP} / \mathrm{bET}$ obtained from the ABI-form device could predict overall and cardiovascular mortality. Therefore, the bPEP/bET might be a useful predictor for overall and cardiovascular mortality among hemodialysis patients, even after including congestive heart failure as a variable.

The prolongation of PEP may be caused by a diminished rate of left ventricular pressure rise during isovolumic contraction. ${ }^{3}$ Increased PEP usually occurred when heart function decreased..$^{5}$ The duration of ET reflects both the velocity and the extent of fiber shortening. In left ventricular decompensation, the extent of fiber shortening is decreased, so a shortened ET is usually noted. ${ }^{4}$ As heart function impairment usually prolongs PEP and shortens ET, the ratio of PEP to ET may enhance the diagnostic value for the identification of left ventricular dysfunction. Our recent study showed that bPEP, bET and bPEP/bET had a significant correlation with left ventricular ejection fraction and bPEP/bET had a higher accuracy in prediction of left ventricular ejection fraction $<50 \%$ than bPEP and bET. ${ }^{6}$ In this study, although bPEP and bET were not predictors of overall and cardiovascular mortality, the bPEP/bET was helpful in the prediction of overall and cardiovascular mortality. Therefore, bPEP/bET may be a useful survival parameter in patients with hemodialysis. This may imply that 
Table 4 Predictors for cardiovascular mortality using Cox proportional hazards model

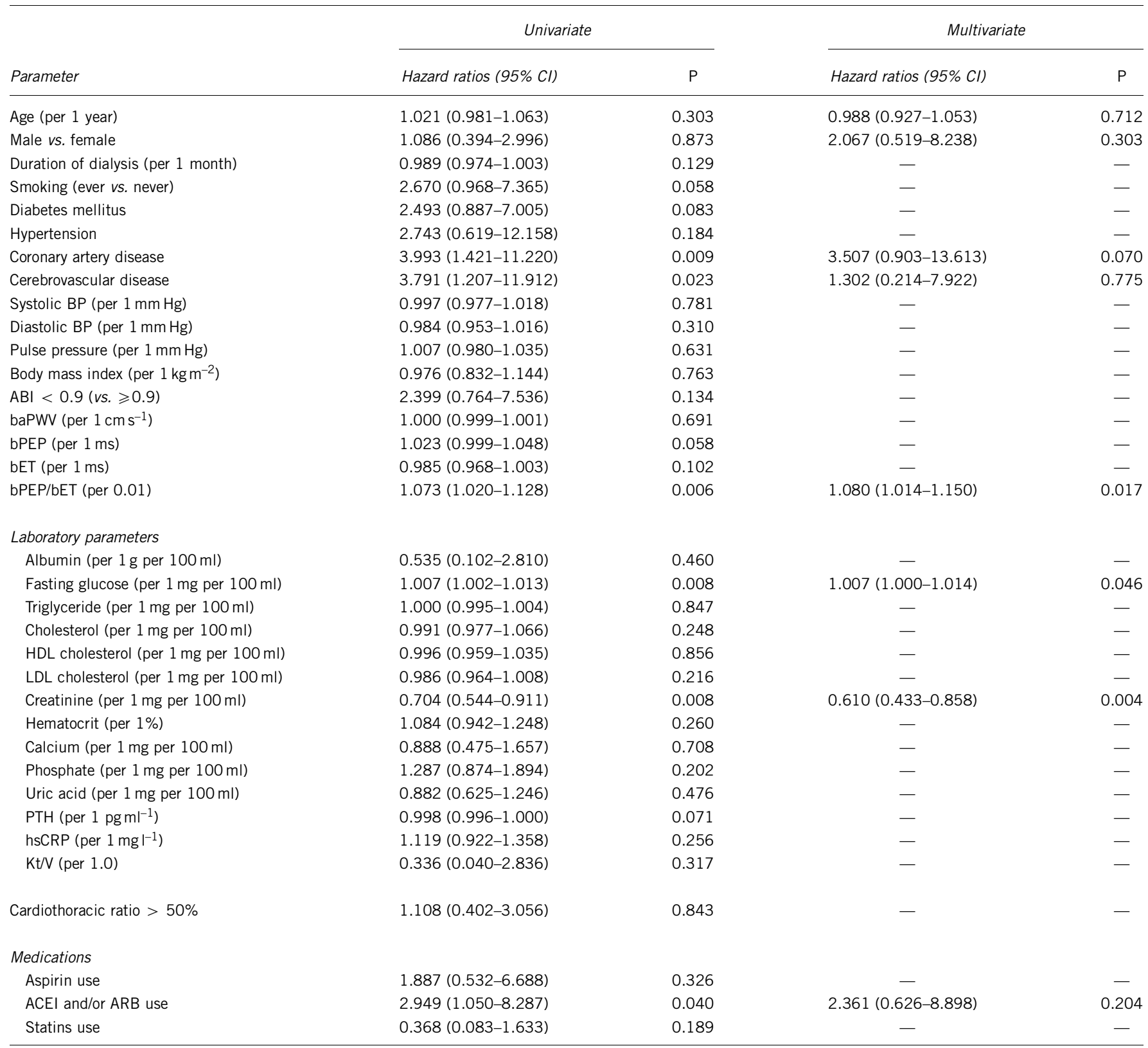

Abbreviations: ABI, ankle-brachial index; ACEI, angiotensin-converting enzyme inhibitor; ARB, angiotensin II receptor blocker; baPWV, brachial-ankle pulse wave velocity; BP, blood pressure; bPEP, brachial pre-ejection period; bET, brachial ejection time; $\mathrm{Cl}$, confidence interval; HDL, high-density lipoprotein; hsCRP, high-sensitivity C-reactive protein; LDL, low-density lipoprotein; PTH, parathyroid hormone.

Values express as hazard ratios and $95 \% \mathrm{Cl}$.

patients with left ventricular systolic dysfunction, indicated by increased bPEP/bET, may be a high-risk group for increased mortality in hemodialysis patients.

Our study subjects were selected from a population of patients receiving regular hemodialysis and their overall health might, therefore, be relatively stable. Therefore, impaired left ventricular systolic function, instead of coronary artery disease, cerebrovascular disease, diabetes or hypertension, might be the chief cause of mortality among these stable patients. ${ }^{11,12}$ Hence, an increase of bPEP/bET, which indicated a decrease in left ventricular systolic function, was a predictor of mortality. However, coronary artery disease, cerebrovascular disease, diabetes and hypertension could not predict mortality independently in this study.
This study revealed that creatinine and PTH levels were lower in patients in the mortality group than in patients in the survival group. Furthermore, after multivariate analysis, serum creatinine level was still associated with overall and cardiovascular mortality. Traditionalrisk factors, such as high blood pressure, obesity and hypercholesterolemia, have an important function in the cardiovascular mortality of the general population. Recently, the concept of reverse epidemiology has been raised, suggesting that low body mass index, low blood pressure, hypocholesterolemia, low low-density lipoprotein cholesterol and low homocysteine level are associated with high cardiovascular mortality and total mortality in dialysis patients. ${ }^{13-15}$ Similar reverse epidemiologic observations have also been found for serum creatinine and PTH levels. ${ }^{13,16,17}$ Reduced intake of calcium, phosphorous and 
protein might lead to low serum creatinine and PTH levels, which usually reflect poor nutritional status and might contribute to high mortality. ${ }^{13,18}$ In this study, patients in the mortality group had lower creatinine and PTH levels and their serum creatinine level was a predictor of overall and cardiovascular mortality (HR, $0.733 ; P=0.029$ and HR, $0.610 ; P=0.004$, respectively) in multivariate analysis. Therefore, malnutrition, as indicated by the low serum creatinine and PTH levels, might have a function in increased mortality in this study.

A long duration of hemodialysis has been reported to be associated with increased mortality in patients with end-stage renal disease, ${ }^{19}$ which might be explained by the vascular calcification caused by alterations in the metabolism of calcium, phosphate and $\mathrm{PTH}$, inflammatory alterations, oxidative stress or hyperhomocysteinemia. ${ }^{20,21}$ Our study showed that patients in the mortality group had shorter hemodialysis duration than survival-group patients did. We further analyzed the determinants of duration of hemodialysis and found that age was negatively correlated with duration of hemodialysis and patients with diabetes mellitus and hypertension had a shorter duration of hemodialysis, which might explain the inconsistent results of earlier studies. ${ }^{22}$ Furthermore, duration of dialysis was not significantly associated with overall mortality after the multivariate Coxregression analysis. Thus, duration of hemodialysis was not a major determinant of overall mortality in this study.

Statins, ACEI, ARB and aspirin have been shown to reduce neointimal proliferation and vascular inflammation. ${ }^{23-26}$ Hemodialysis patients are usually prescribed many of these medications for cardiovascular causes. In this study, patients in the mortality group had a higher percentage of having received aspirin or ACEI and/or ARB therapy. Further analysis found that the use of aspirin or ACEI and/or ARB was more frequent among patients with coronary artery disease (26.7 vs. $6.6 \%, P<0.001$ and 31.7 vs. $15.2 \% ; P=0.007$, respectively), which might partially explain the greater use of aspirin or ACEI and/or ARB in our patients in the mortality group. Furthermore, the use of aspirin or ACEI and/or ARB was not significantly associated with overall mortality after multivariate Cox-regression analysis. Thus, treatment with aspirin or ACEI and/or ARB was not a major determinant of overall mortality in this study.

The measurement of bPEP and bET may be influenced by hemodialysis. ${ }^{27,28}$ To avoid interference from hemodialysis in this study, bPEP and bET were measured before hemodialysis in all study subjects. In addition, because of ethical reasons, bPEP and bET were only measured on the arm, without blood access. Hence, in this study, the bPEP and bET were obtained from the right and left brachium in 168 and 44 patients, respectively. The bET represented the ET of the left ventricle. Therefore, bET should be unchanged whether it was obtained from the right or the left brachium. The bPEP was calculated by subtracting the bET from the $\mathrm{QS}_{2}$, so it should be also unchanged whether it was measured from the right or the left brachium. Hence, $\mathrm{bPEP} / \mathrm{bET}$ is likely to be unchanged when obtained from the right or left brachium.

There were several limitations to this study. The study subjects were enrolled from only one regional hospital and the selection of patients was relatively restricted. Therefore, generality of the results was limited. In addition, we did not further validate our finding with invasive methodology or noninvasive echocardiographic parameters.

In conclusion, our results show that bPEP/bET, a surrogate of left ventricular systolic function, obtained from the ABI-form device can predict overall and cardiovascular mortality in patients with hemodialysis. Screening hemodialysis patients by means of bPEP/bET may help to identify a high-risk group for increased mortality.

\section{CONFLICT OF INTEREST}

The authors declare no conflict of interest.

\section{ACKNOWLEDGEMENTS}

The research presented in this article is supported by the grant from Kaohsiung Municipal Hsiao-Kang Hospital (kmhk-98-015), Kaohsiung Medical University, Kaohsiung, Taiwan.

1 Mallick NP, Jones E, Selwood N. The European (European Dialysis and Transplantation Association-European Renal Association) Registry. Am J Kidney Dis 1995; 25: $176-187$.

2 Shinzato T, Nakai S, Akiba T, Yamagami S, Yamazaki C, Kitaoka T, Kubo K, Maeda K, Morii H. Report of the annual statistical survey of the Japanese Society for Dialysis Therapy in 1996. Kidney Int 1999; 55: 700-712.

3 Lewis RP, Rittogers SE, Froester WF, Boudoulas H. A critical review of the systolic time intervals. Circulation 1977; 56: 146-158.

4 Garrard Jr CL, Weissler AM, Dodge HT. The relationship of alterations in systolic time intervals to ejection fraction in patients with cardiac disease. Circulation 1970; 42: 455-462.

5 Veyrat C, Larrazet F, Pellerin D. Renewed interest in preejectional isovolumic phase: new applications of tissue Doppler indexes: implications to ventricular dyssynchrony. Am J Cardiol 2005; 96: 1022-1030.

6 Su HM, Lin TH, Lee CS, Lee HC, Chu CY, Hsu PC, Voon WC, Lai WT, Sheu SH. Myocardial performance index derived from brachial-ankle pulse wave velocity: a novel and feasible parameter in evaluation of cardiac performance. Am J Hypertens 2009; 22: 871-876.

7 Yamashina A, Tomiyama H, Takeda K, Tsuda H, Arai T, Hirose K, Koji Y, Hori S, Yamamoto $Y$. Validity, reproducibility, and clinical significance of noninvasive brachial-ankle pulse wave velocity measurement. Hypertens Res 2002; 25: 359-364.

8 Tomiyama H, Yamashina A, Arai T, Hirose K, Koji Y, Chikamori T, Hori S, Yamamoto Y, Doba N, Hinohara S. Influences of age and gender on results of noninvasive brachialankle pulse wave velocity measurement-a survey of 12517 subjects. Atherosclerosis 2003; 166: 303-309.

9 Yokoyama H, Shoji T, Kimoto E, Shinohara K, Tanaka S, Koyama H, Emoto M, Nishizawa Y. Pulse wave velocity in lower-limb arteries among diabetic patients with peripheral arterial disease. J Atheroscler Thromb 2003; 10: 253-258.

10 Gotch FA. Evolution of the single-pool urea kinetic model. Semin Dial 2001; 14: 252-256.

11 Macchia A, Levantesi G, Franzosi MG, Geraci E, Maggioni AP, Marfisi R, Nicolosi GL, Schweiger C, Tavazzi L, Tognoni G, Valagussa F, Marchioli R. GISSI-prevenzione investigators. Left ventricular systolic dysfunction, total mortality, and sudden death in patients with myocardial infarction treated with n-3 polyunsaturated fatty acids.. Eur J Heart Fail 2005; 7: 904-909.

12 Hobbs FD, Roalfe AK, Davis RC, Davies MK, Hare R. Midlands Research Practices Consortium (MidReC). Prognosis of all-cause heart failure and borderline left ventricular systolic dysfunction: 5 year mortality follow-up of the Echocardiographic Heart of England Screening Study (ECHOES). Eur Heart J 2007; 28: 1128-1134.

13 Kopple JD. The phenomenon of altered risk factor patterns or reverse epidemiology in persons with advanced chronic kidney failure. Am J Clin Nutr 2005; 81: 1257-1266.

14 Levin NW, Handelman GJ, Coresh J, Port FK, Kaysen GA. Reverse epidemiology: a confusing, confounding, and inaccurate term. Semin Dial 2007; 20: 586-592.

15 Mistrik E, Blaha V, Dusilova Sulkova S, Sobotka L. The MIAC (malnutrition, inflammation, atherosclerosis, calcification) syndrome. Vnitr Lek 2007; 53: 1092-1099.

16 Kalantar-Zadeh K, Stenvinkel P, Pillon L, Kopple JD. Inflammation and nutrition in renal insufficiency. Adv Ren Replace Ther 2003; 10: 155-169.

17 Walser M. Creatinine excretion as a measure of protein nutrition in adults of varying age. JPEN J Parenter Enteral Nutr 1987; 11: 73S-78S.

18 Guh JY, Chen HC, Chuang HY, Huang SC, Chien LC, Lai YH. Risk factors and risk for mortality of mild hypoparathyroidism in hemodialysis patients. Am J Kidney Dis 2002; 39: 1245-1254.

19 Qunibi WY, Nolan CA, Ayus JC. Cardiovascular calcification in patients with end-stage renal disease: a century-old phenomenon. Kidney Int Supp/ 2002; 82: S73-S80.

20 O'Hare A, Johansen K. Lower-extremity peripheral arterial disease among patients with end-stage renal disease. J Am Soc Nephrol 2001; 12: 2838-2847.

21 Webb AT, Franks PJ, Reaveley DA, Greenhalgh RM, Brown EA. Prevalence of intermittent claudication and risk factors for its development in patients on renal replacement therapy. Eur J Vasc Surg 1993; 7: 523-527.

22 Iseki K, Tozawa M, Takishita S. Effect of the duration of dialysis on survival in a cohort of chronic haemodialysis patients. Nephrol Dial Transplant 2003; 18 : 782-787. 
23 Dichtl W, Dulak J, Frick M, Alber HF, Schwarzacher SP, Ares MP, Nilsson J, Pachinger $O$, Weidinger $\mathrm{F}$. HMG-COA reductase inhibitors regulate inflammatory transcription factors in human endothelial and vascular smooth muscle cells. Arterioscler Thromb Vasc Biol 2003; 23: 58-63.

24 Gradzki R, Dhingra RK, Port FK, Roys E, Weitzel WF, Messana JM. Use of ACE inhibitors is associated with prolonged survival of arteriovenous grafts. Am J Kidney Dis 2001; 38: 1240-1244.

25 Horiuchi M, Cui TX, Li Z, Li JM, Nakagami H, Iwai M. Fluvastatin enhances the inhibitory effects of a selective angiotensin II type 1 receptor blocker, valsartan, on vascular neointimal formation. Circulation 2003; 107: 106-112.
26 Torsney E, Mayr U, Zou Y, Thompson WD, Hu Y, Xu Q. Thrombosis and neointima formation in vein grafts are inhibited by locally applied aspirin through endothelial protection. Circ Res 2004; 94: 1466-1473.

27 Su HM, Chang JM, Lin FH, Chen SC, Voon WC, Cheng KH, Wang CS, Lin TH, Lai WT, Sheu SH. Influence of different measurement time points on brachial-ankle pulse wave velocity and ankle-brachial index in hemodialysis patients. Hypertens Res 2007; 30: 965-970.

28 Su HM, Lin TH, Voon WC, Chen HC, Lin FH, Kuo MC, Lee KT, Chu CS, Lai WT, Sheu SH. Impact of preload alterations by haemodialysis on the Tei index: comparison between pulsed flow and tissue Doppler analyses. Acta Cardiol 2007; 62: 25-30. 